\title{
Space-time description of strong-field ionization and high-order-harmonic generation
}

\author{
C. Granados ${ }^{1,2}$ and L. Plaja ${ }^{1}$ \\ ${ }^{1}$ Grupo de Investigación en Óptica Extrema (GIOE), Universidad de Salamanca, E-37008 Salamanca, Spain \\ ${ }^{2}$ Instituut voor Kern- en Stralingsfysica, KU Leuven, University of Leuven, B-3001 Leuven, Belgium
}

(Received 11 December 2013; published 24 February 2014)

\begin{abstract}
We develop the spatiotemporal description of matter-field interaction within the strong-field approximation. We show that the space-time form of the ionized wave function has analogies with the diffraction phenomenon, allowing for the definition of two different regimes: Fresnel and Fraunhofer. We demonstrate that the standard saddle-point analysis corresponds to the paraxial approximation of the Fraunhofer case. The Fresnel number therefore appears as a useful parameter to characterize the validity of the saddle-point approach. We give a closed formula for the ionized wave function beyond the standard saddle-point analysis that takes the form of a chirped Volkov wave. We apply our results to the study of high-order-harmonic generation, demonstrating that the saddle-point approximation breaks down for extended systems, i.e., when the Fresnel number approaches or is above the unity. As a simple example, we analyze the harmonic generation of dissociating $H_{2}^{+}$and demonstrate the Fresnel number as a useful parameter to determine the accuracy of the semiclassical saddle-point approach.
\end{abstract}

DOI: 10.1103/PhysRevA.89.023428

PACS number(s): $32.80 . \mathrm{Fb}, 32.80 . \mathrm{Rm}, 42.65 . \mathrm{Ky}$

\section{INTRODUCTION}

Laser technology has reached a point where even smallsized laboratories can host intense sources. Therefore, intenselaser physics offers an excellent playground for developing our understanding of quantum physics beyond the perturbative limit, in which the experimental validation is regularly available. Since many of the interesting phenomena in this field are related to the dynamics of single electrons, the theoretical treatment is greatly simplified and can be treated using exact solution of the one-particle time-dependent Schrödinger equation (TDSE). Nevertheless, for applications involving macroscopic targets, the numerical computation of the exact wave function at every target point is time-consuming; therefore there is a practical necessity to develop approximated models. In addition, semianalytical models provide a physical picture which is hidden in the exact computations.

A recurring approximation in strong field physics is the strong-field approximation (SFA), which consists of three assumptions: First, the interaction with the ground-state electron is assumed to conduct solely to ionization (i.e., no bound-state excitations are considered); second, the ionized electron is assumed to be a free particle in the electromagnetic field, neglecting the Coulomb interaction with the parent ion; and third, the ionized electron is assumed to remain in the continuum during the rest of the interaction. The SFA was first used by Keldysh et al. [1-3] to study the photoelectron spectrum and later was applied by Lewenstein to high-order harmonic generation $[4,5]$.

SFA theories are naturally developed in momentum space, as the continuum free-electron approximation allows for the efficient use of the Volkov basis [6], i.e., the plane-wave states of a free electron in an electromagnetic field. However, spacetime approaches have been used in other non-SFA treatments of intense-laser matter interaction, as for instance the adiabatic approach in Ref. [7]. Except for the case of a monochromatic field, there is no analytical solution for the time evolution of the SFA wave function. Generally the solution is found semianalytically, resorting to the saddle-point approximation to compute the momentum-space integrals and, optionally, also the integral over all ionization times.

In this paper we develop a position-space approach for the strong-field problem that constitutes a complementary view to the momentum-space description mentioned above. Our viewpoint allows us to develop an analogy with the optical scalar theory of diffraction. We derive some practical results from this such as, for instance, a quantitative test for the validity of the saddle-point approach (frequently used in the standard SFA derivation), and explicit forms for the ionized wave packet, more precise than the usual SFA plane-wave assumption.

The paper is organized as follows: We start giving the general formula for the spatial form of the wave function of the electron in the continuum, in terms of the interference of the wave packets ionized at previous instants of time. The computation of each of these elementary contributions is not trivial, as it contains an integral over space. Next, we develop an analogy with optical scalar diffraction theory that will allow us to define a Fresnel number $N_{F}$ and, therefore to find the regimes for the ionized wave function analogous to the far field $\left(N_{F} \ll 1\right)$ and near field $\left(N_{F} \geqslant 1\right)$ in diffractive optics. The far-field regime is found to be a valid description of the ionized wave function from small systems (atoms and small molecules) and/or for sufficient large excursion times of the ionized electron in the continuum. For this far-field case, we derive a compact expression for the ionized wave function, which is more general than the Volkov plane wave. Following the optical analogy, we demonstrate that the standard SFA formulation, developed in momentum space and using the saddle-point approximation (SPA), corresponds to the paraxial case of the far-field diffraction, and therefore, the SFA-SPA wave function is a particular case of our more general SFA wave function for the ionized electron in the far-field regime. The practical implication of this is that we can propose the Fresnel number $N_{F}$ as a quantitative measure for the validity of the standard SFA-SPA approach. In a second part of the paper, we use our result to compute high-order harmonic generation (HHG). We demonstrate that, in the 
paraxial far-field regime, the formulation of HHG leads to the the Lewenstein's saddle-point approach [4]. We show that, while harmonic generation in small systems (atoms and small molecules) fulfills the paraxial far-field condition, systems larger than a few tens of atomic units require the exact (i.e., non-saddle-point) SFA computation. We conclude the paper with the analysis of harmonic generation of a dissociating $\mathrm{H}_{2}^{+}$molecule. This system is especially suitable to show the applicability of our results, since by changing the distances between the ions we can explore the breakdown of the paraxial far-field regime, or equivalently the saddle-point approach, in comparison with the exact evaluation of the SFA harmonic spectrum.

\section{SPACE-TIME FORM OF THE IONIZED WAVE FUNCTION}

Our aim is to develop the solution of the Schrödinger equation, for the single-active electron and in the strong-field approximation (SFA), using space-time coordinates instead of the usual momentum space approach,

$$
i \hbar \frac{\partial}{\partial t}|\psi(t)\rangle=\left[H_{A}+V_{F}(t)\right]|\psi(t)\rangle,
$$

where $\hat{H}_{A}=\hat{\pi}^{2} / 2 m+V_{C}$ is the atomic Hamiltonian $\left(V_{C}\right.$ is the Coulomb potential and $\hat{\boldsymbol{\pi}}$ is the linear momentum operator). $V_{F}(t)=-q \mathbf{r} \cdot \mathbf{E}(t)$ describes the interaction with the electromagnetic field in the length gauge ( $q$ is the electron charge and $\mathbf{E}(t)$ is the electric field in dipole approximation).

The solution of Eq. (1) can be written as $|\psi(t)\rangle=\left|\phi_{0}(t)\right\rangle+$ $|\tilde{\psi}(t)\rangle$, where

$$
\left|\phi_{0}(t)\right\rangle=\exp \left[-i \epsilon_{0}\left(t-t_{0}\right) / \hbar\right]\left|\phi_{0}\right\rangle
$$

is the field-free evolution of the initial state $\left|\phi_{0}\right\rangle$, in which we assume a bound state of the atom $H_{A}\left|\phi_{0}\right\rangle=\epsilon_{0}\left|\phi_{0}\right\rangle$, and where

$$
|\tilde{\psi}(t)\rangle=\frac{1}{\hbar} \int_{t_{0}}^{t} G^{+}\left(t, t_{i}\right) V_{F}\left(t_{i}\right)\left|\phi_{0}\left(t_{i}\right)\right\rangle d t_{i}
$$

describes the wave function perturbed by the field. $G^{+}\left(t, t_{i}\right)$ is the propagator describing the exact evolution from an initial ionization time $t_{i}$ to any final time $t$ and $|\psi(t)\rangle=$ $i G^{+}\left(t, t_{i}\right)\left|\psi\left(t_{i}\right)\right\rangle$. The SFA replaces $G^{+}\left(t, t_{i}\right)$ by the propagator $G_{F}^{+}\left(t, t_{i}\right)$ of a free electron evolving solely under the influence of the electric field, associated with the Hamiltonian $\hat{H}_{F}=$ $\hat{\boldsymbol{\pi}}^{2} / 2 m+V_{F}(t)$, and therefore (3) is approximated to

$$
|\tilde{\psi}(t)\rangle=\frac{1}{\hbar} \int_{t_{0}}^{t} G_{F}^{+}\left(t, t_{i}\right) V_{F}\left(t_{i}\right)\left|\phi_{0}\left(t_{i}\right)\right\rangle d t_{i} .
$$

As shown in Appendix A, the spatial form of $|\tilde{\psi}(t)\rangle$ can be found from Eq. (4) as

$$
\begin{aligned}
\tilde{\psi}(\mathbf{r}, t)= & \frac{1}{i \hbar} \int_{t_{0}}^{t} \int\left[\frac{m}{2 \pi i \hbar\left(t-t_{i}\right)}\right]^{3 / 2} e^{\frac{i}{\hbar} S_{c l}\left(\mathbf{r}, t, \mathbf{r}_{i}, t_{i}\right)} \\
& \times\left\langle\mathbf{r}_{i}\left|V_{F}\left(t_{i}\right)\right| \phi_{0}\left(t_{i}\right)\right\rangle d \mathbf{r}_{i} d t_{i},
\end{aligned}
$$

where $\tilde{\psi}(\mathbf{r}, t) \equiv\langle\mathbf{r} \mid \tilde{\psi}(t)\rangle, \mathbf{r}$ and $\mathbf{r}_{i}$ are the electron's coordinates at times $t$ and $t_{i}$, respectively, and $S_{c l}$ corresponds to the action associated to the classical trajectory of a free electron in the electromagnetic field, starting at $\mathbf{r}_{i}$ at time $t_{i}$ and reaching $\mathbf{r}$ at time $t$.

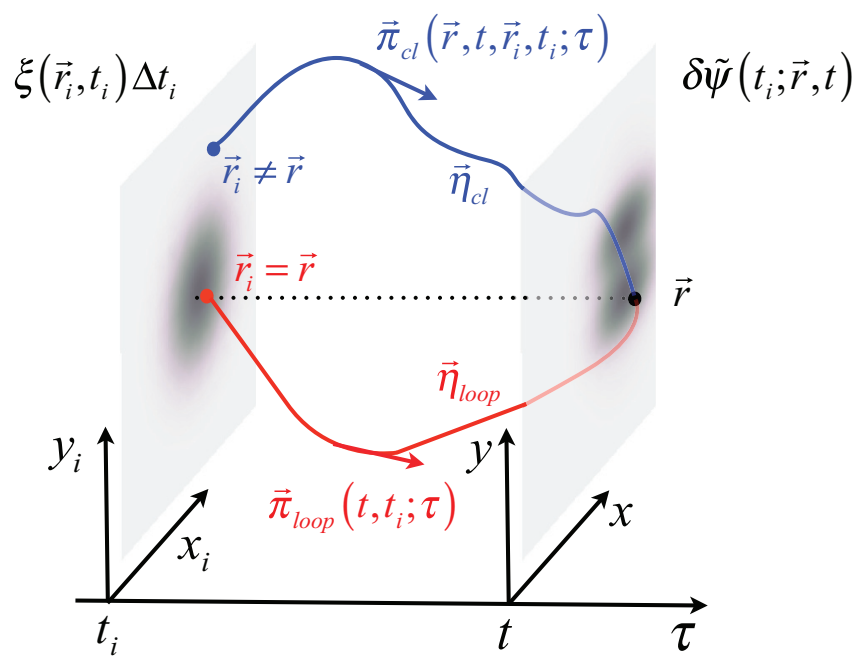

FIG. 1. (Color online) Scheme of the wave function propagation from the initial ionization time $t_{i}$ to an arbitrary final time $t$. Following Eq. (7), the amplitude probability at each point of the evolving wave function results from the interference of the classical trajectories starting at any point $\mathbf{r}_{i}$ of the initial wave function, with a kinetic momentum given by Eq. (10). The loop trajectory, highlighted in red (gray), corresponds to the special case in which the electron returns to its initial position $\left(\mathbf{r}=\mathbf{r}_{i}\right)$. For clarity, the wave functions are represented only in two dimensions.

The SFA wave function (5) can be understood as composed of elementary contributions associated to every ionization time

$$
\tilde{\psi}(\mathbf{r}, t)=\sum_{t_{i}} \delta \tilde{\psi}\left(t_{i} ; \mathbf{r}, t\right)
$$

where

$$
\begin{aligned}
\delta \tilde{\psi}\left(t_{i} ; \mathbf{r}, t\right)= & \left(\frac{m}{2 \pi i \hbar} \frac{1}{t-t_{i}}\right)^{3 / 2} \\
& \times \int e^{\frac{i}{\hbar} S_{c l}\left(\mathbf{r}, t, \mathbf{r}_{i}, t_{i}\right)} \xi\left(\mathbf{r}_{i}, t_{i}\right) d \mathbf{r}_{i} \Delta t_{i}
\end{aligned}
$$

represents the time-evolving wave function of an electron ionized during the differential time interval from $t_{i}$ to $t_{i}+\Delta t_{i}$. The quantity

$$
\xi\left(\mathbf{r}_{i}, t_{i}\right) \equiv(1 / i \hbar)\left\langle\mathbf{r}_{i}\left|V_{F}\left(t_{i}\right)\right| \phi_{0}\left(t_{i}\right)\right\rangle
$$

can be interpreted as the rate of probability amplitude injection from the bound state into the continuum; i.e., $\xi\left(\mathbf{r}_{i}, t_{i}\right) \Delta t_{i}$ is the wave function that appears in the continuum during the ionization time interval from $t_{i}$ to $t_{i}+\Delta t_{i}$.

Figure 1 shows a simplified sketch of the situation described by Eq. (7): At any time $t$ the wave function, evolved from the ionization event at $t_{i}$, is the superposition of the contributions of all classical trajectories connecting the final coordinate $\mathbf{r}$ with every possible starting point $\mathbf{r}_{i}$ at $t_{i}$. Each trajectory, $\boldsymbol{\eta}_{c l}$, is defined by its starting point and path shape, given by the time integral of the kinetic momentum

$$
\begin{aligned}
\boldsymbol{\pi}_{c l}\left(\mathbf{r}, t, \mathbf{r}_{i}, t_{i}, ; \tau\right)= & -\frac{q}{c} \mathbf{A}(\tau)+\frac{1}{t-t_{i}}\left[m\left(\mathbf{r}-\mathbf{r}_{i}\right)\right. \\
& \left.+\frac{q}{c} \int_{t_{i}}^{t} \mathbf{A}\left(\tau^{\prime}\right) d \tau^{\prime}\right] .
\end{aligned}
$$


Therefore

$$
\boldsymbol{\eta}_{c l}\left(\mathbf{r}, t, \mathbf{r}_{i}, t_{i} ; \tau\right)=\mathbf{r}_{i}+\frac{1}{m} \int_{t_{i}}^{\tau} \pi_{c l}\left(\mathbf{r}, t, \mathbf{r}_{i}, t_{i} ; \tau^{\prime}\right) d \tau^{\prime} .
$$

The explicit form for the semiclassical action $S_{c l}\left(r, t, r_{i}, t_{i} s\right)$ in (7) is (see Appendix A)

$$
\begin{aligned}
S_{c l}\left(\mathbf{r}, t, \mathbf{r}_{i}, t_{i}\right)= & -\frac{1}{2 m} \int_{t_{i}}^{t} \pi_{c l}^{2}\left(\mathbf{r}, t, \mathbf{r}_{i}, t_{i}, ; \tau\right) d \tau \\
& +\pi_{c l}\left(\mathbf{r}, t, \mathbf{r}_{i}, t_{i}, ; t\right) \cdot \mathbf{r}-\boldsymbol{\pi}_{c l}\left(\mathbf{r}, t, \mathbf{r}_{i}, t_{i}, ; t_{i}\right) \cdot \mathbf{r}_{i} .
\end{aligned}
$$

Among all possible trajectories represented in Eq. (10), we refer to those paths for which the electron returns to the initial position as loop trajectories. The kinetic momentum in this case is given by imposing the condition $\mathbf{r}=\mathbf{r}_{i}$ to Eq. (10), leading to

$$
\boldsymbol{\pi}_{\text {loop }}\left(t, t_{i} ; \tau\right) \equiv-\frac{q}{c}\left[\mathbf{A}(\tau)-\frac{1}{t-t_{i}} \int_{t_{i}}^{t} \mathbf{A}(\tau) d \tau\right],
$$

Note that since $\pi_{\text {loop }}$ does not depend on the electron coordinates, the shape of the loop trajectory is a characteristic of the dynamics between $t_{i}$ and $t$ : All other trajectories, where the electron does not return to the initial position, can be considered as variations of the loop case, since from Eqs. (10) and (12) we have

$$
\boldsymbol{\pi}_{c l}\left(\mathbf{r}, t, \mathbf{r}_{i}, t_{i} ; \tau\right)=m \frac{\mathbf{r}-\mathbf{r}_{i}}{t-t_{i}}+\boldsymbol{\pi}_{\text {loop }}\left(t, t_{i} ; \tau\right) .
$$

This relation is of practical utility, since it shows explicitly the dependence of the dynamics of the ionized electron with the initial and final coordinates. In particular the classical action (11) can be rewritten as

$$
\begin{aligned}
S_{c l}\left(\mathbf{r}, t, \mathbf{r}_{i}, t_{i}\right)= & -\frac{1}{2 m} \int_{t_{i}}^{t} \pi_{\text {loop }}^{2}\left(t, t_{i} ; \tau\right) d \tau+\frac{m}{2} \frac{\left(\mathbf{r}-\mathbf{r}_{i}\right)^{2}}{t-t_{i}} \\
& +\boldsymbol{\pi}_{\text {loop }}\left(t, t_{i} ; t\right) \cdot \mathbf{r}-\boldsymbol{\pi}_{\text {loop }}\left(t, t_{i} ; t_{i}\right) \cdot \mathbf{r}_{i} .
\end{aligned}
$$

Therefore, the exact SFA wave function (7) can be written

$$
\begin{aligned}
\delta \tilde{\psi}\left(t_{i} ; \mathbf{r}, t\right)= & \left(\frac{m}{2 \pi i \hbar} \frac{1}{t-t_{i}}\right)^{3 / 2} e^{-\frac{i}{2 m \hbar} \int_{t_{i}}^{t} \pi_{\text {loop }}^{2}\left(t, t_{i} ; \tau\right) d \tau} \\
& \times e^{\frac{i}{\hbar} \pi_{\text {loop }}\left(t, t_{i} ; t\right) \cdot \mathbf{r}} \int e^{i \frac{m}{2 \hbar} \frac{\left(\mathbf{r}-\mathbf{r}_{i}\right)^{2}}{t-t_{i}}} e^{-\frac{i}{\hbar} \pi_{\text {loop }}\left(t, t_{i} ; t_{i}\right) \cdot \mathbf{r}_{i}} \\
& \times \xi\left(\mathbf{r}_{i}, t_{i}\right) d \mathbf{r}_{i} \Delta t_{i}
\end{aligned}
$$

\section{ANALOGY WITH DIFFRACTION THEORY}

In this section we use the above definitions to develop further the spatiotemporal picture of the strong field-atom interaction. In the first place, we introduce an analogy with the optical scalar diffraction theory. This will allow us to represent the evolution of the ionized wave function as the formation of the diffraction pattern of light after passing through an aperture. The excursion time $t-t_{i}$, properly dimensioned, will assume a role analogous to the distance between the diffraction aperture and the screen where the diffraction pattern is projected. The association will suggest the definition of two different regimes for the ionized wave function, corresponding to short excursion times (near field, or Fresnel diffraction) and long excursions (far field, or Fraunhofer diffraction).

The analogy follows from rewritting Eq. (15) as

$$
\begin{aligned}
& e^{-\frac{i}{\hbar} \pi_{\text {loop }}\left(t, t_{i} ; t\right) \cdot \mathbf{r}} \delta \tilde{\psi}\left(t_{i} ; \mathbf{r}, t\right) \\
& =\left(\frac{m}{2 \pi i \hbar} \frac{1}{t-t_{i}}\right)^{3 / 2} e^{-\frac{i}{\hbar}\left\langle T_{\text {loop }}\right\rangle\left(t-t_{i}\right)} \\
& \quad \times \int e^{\frac{i}{\hbar} \frac{m}{2} \frac{\left(\mathbf{r}-\mathbf{r}_{i}\right)^{2}}{t-t_{i}}} e^{-\frac{i}{\hbar} \pi_{\text {loop }}\left(t, t_{i} ; t_{i}\right) \cdot \mathbf{r}_{i}} \xi\left(\mathbf{r}_{i}, t_{i}\right) d \mathbf{r}_{i} \Delta t_{i},
\end{aligned}
$$

where $\left\langle T_{\text {loop }}\right\rangle=1 /\left[2 m\left(t-t_{i}\right)\right] \int_{t_{i}}^{t} \pi_{\text {loop }}^{2}\left(t, t_{i} ; \tau\right) d \tau$ is the mean kinetic energy of the loop trajectory. Equation (16) can be cast in the form of a three-dimensional generalization of the Fresnel diffraction integral [8]:

$$
U(\mathbf{r}, \zeta)=\frac{e^{-i k_{\zeta} \zeta}}{\left(i \lambda_{\zeta} \zeta\right)^{3 / 2}} \int e^{i \frac{k_{\zeta}}{2 \zeta}\left(\mathbf{r}-\mathbf{r}_{i}\right)^{2}} U\left(\mathbf{r}_{i}, 0\right) d \mathbf{r}_{i}
$$

using the following definitions:

(i) The distance between the aperture plane and the screen is given by $\zeta$, which is defined in terms of the excursion time of the ionized wave function, $t-t_{i}$, as $\zeta=\left(t-t_{i}\right) \sqrt{\left\langle T_{\text {loop }}\right\rangle / m}$. Therefore, longer excursion times correspond to a diffraction setup with a larger distance between the aperture and the screen.

(ii) $k_{\zeta}$ is the wave number of the diffracted field, which is defined as $k_{\zeta}=(1 / \hbar) \sqrt{m\left\langle T_{\text {loop }}\right\rangle}$, leading to the definition of $\lambda_{\zeta}=2 \pi / k_{\zeta}$.

(iii) $U\left(\mathbf{r}_{i}, 0\right)=e^{-\frac{i}{\hbar} \pi_{\text {loop }}\left(t, t_{i} ; t_{i}\right) \cdot \mathbf{r}_{i}} \xi\left(\mathbf{r}_{i}, t_{i}\right) \Delta t_{i}$ corresponds to the distribution of the optical field at the aperture and relates to the wave function at the ionization time. Since the wave function is a tridimensional object, the integral at the aperture in Eq. (17) involves three dimensions instead of two, which is the usual in the optical scalar diffraction theory. Without lost of generality, we consider our reference frame located at the mean position of the wave function at ionization time, i.e., $\left\langle\mathbf{r}_{i}\right\rangle_{\xi}=0$.

(iv) The field at the image plane is given by the wave function at time $t$, as $U(\mathbf{r}, \zeta)=e^{-\frac{i}{\hbar} \pi_{\text {loop }}\left(t, t_{i} ; t\right) \cdot \mathbf{r}} \delta \tilde{\psi}\left(t_{i} ; \mathbf{r}, t\right)$.

The parallelism with optical diffraction provides a framework that can be exploited to give some practical results. For this, let us expand the argument of the Gaussian exponential in Eq. (17) and rewrite

$$
U(\mathbf{r}, \zeta)=\frac{e^{-i k_{\zeta} \zeta}}{\left(i \lambda_{\zeta} \zeta\right)^{3 / 2}} e^{i \pi N_{F} \frac{r^{2}}{\left(r r_{0}^{2}\right.}} \int e^{i \frac{k_{\zeta}}{\zeta} \mathbf{r} \cdot \mathbf{r}_{i}} e^{i \pi N_{F} \frac{r_{i}^{2}}{\left\langle\left. r\right|_{0} ^{2}\right.}} U\left(\mathbf{r}_{i}, 0\right) d \mathbf{r}_{i},
$$

where $\langle r\rangle_{0}$ is the size of the wave function at the instant of ionization (approximately the mean radius of the bound state) that plays the role of a characteristic size of the diffraction aperture. $N_{F}$ is the Fresnel number

$$
N_{F} \equiv \frac{1}{2 \pi} \frac{k_{\zeta}}{\zeta}\langle r\rangle_{0}^{2}=\frac{m}{2 \pi \hbar} \frac{\langle r\rangle_{0}^{2}}{t-t_{i}} .
$$

The condition $N_{F} \ll 1$ corresponds to the far-field or Fraunhofer regime of diffraction. Since typically $\left|\mathbf{r}_{i}\right| \sim\langle r\rangle_{0}$, if $N_{F} \ll 1$ the exponential with the quadratic argument inside the integral of Eq. (18) can be neglected and the field at the screen 
corresponds to the Fourier transform of the field at the aperture (Fraunhofer integral). In our analogy, the Fraunhofer regime is attained for small-size wave functions at ionization times and/or for long excursion times. This is a typical situation in, for instance, strong-field ionization of atoms and small molecules.

Using Eq. (18) in the far-field regime $\left(N_{F} \ll 1\right)$ and transforming back $U\left(\mathbf{r}_{i}, 0\right)$ and $U(\mathbf{r}, \zeta)$ in terms of wave functions, we retrieve a compact expression for the time evolution of the wave function ionized at $t_{i}$ and observed at $t$

$$
\begin{aligned}
& \delta \tilde{\psi}_{F F}\left(t_{i} ; \mathbf{r}, t\right) \\
& =\left(\frac{m}{i \hbar} \frac{1}{t-t_{i}}\right)^{3 / 2} e^{-\frac{i}{2 m \hbar} \int_{t_{i}}^{t} \pi_{\text {loop }}^{2}\left(t, t_{i} ; \tau\right) d \tau} \\
& \quad \times e^{\frac{i}{\hbar}\left[\pi_{\text {loop }}\left(t, t_{i} ; t\right)+\frac{m}{2} \frac{\mathbf{r}}{t-t_{i}}\right] \cdot \mathbf{r}} \Xi\left[\pi_{\text {loop }}\left(t, t_{i} ; t_{i}\right)+m \frac{\mathbf{r}}{t-t_{i}}, t_{i}\right] \Delta t_{i},
\end{aligned}
$$

where $\boldsymbol{\Xi}\left(\boldsymbol{\pi}, t_{i}\right)$ is the Fourier transform of $\xi\left(\mathbf{r}_{i}, t_{i}\right)$,

$$
\Xi\left(\boldsymbol{\pi}, t_{i}\right)=\frac{1}{(2 \pi)^{3 / 2}} \int e^{-\frac{i}{\hbar} \pi \cdot \mathbf{r}_{i}} \xi\left(\mathbf{r}_{i}, t_{i}\right) d \mathbf{r}_{i} .
$$

The ionized wave function (20) can be interpreted as a chirped Volkov wave, where the electron's kinetic momentum varies as a function of the spatial coordinate. The departure from the ordinary Volkov wave becomes significant at large distances from the ionization spot, and therefore, Eq. (20) offers a better description of the free electron in the field wave function (compared with the standard SPA) in situations where the ionized electron is far from the parent ion, which is a situation that is relevant, for instance, in interion electron transfer and collisional excitation [9].

The far-field equation (20) allows for a further simplification if we are interested in describing the ionized wave function at the neighborhood of the ionization spot. The corresponding condition for this is $r^{2} \ll\langle r\rangle_{0}^{2} / N_{F}$, that corresponds to diffraction optics in the paraxial far-field regime $\left(r / \zeta \ll \lambda_{\zeta} / r\right)$. In this case Eq. (20) is simplified to

$$
\begin{aligned}
\delta \tilde{\psi}_{\mathrm{SPA}}\left(t_{i} ; \mathbf{r}, t\right)= & \left(\frac{m}{i \hbar} \frac{1}{t-t_{i}}\right)^{3 / 2} e^{-\frac{i}{2 m \hbar} \int_{t_{i}}^{t} \pi_{\text {loop }}^{2}\left(t, t_{i} ; \tau\right) d \tau} \\
& \times e^{\frac{i}{\hbar}\left[\pi_{\text {loop }}\left(t, t_{i} ; t\right)\right] \cdot \mathbf{r}} \Xi\left[\boldsymbol{\pi}_{\text {loop }}\left(t, t_{i} ; t_{i}\right), t_{i}\right] \Delta t_{i},
\end{aligned}
$$

that corresponds to the plane-wave solution obtained when developing SFA theory in momentum space and using the saddlepoint approximation (SPA); see Appendix B. Therefore, we label it according to this equivalence. Our approach demonstrates, therefore, that the standard SFA approach, involving SPA and developed in momentum space, is equivalent to the paraxial far-field regime. Therefore, SFA-SPA approaches are found to be valid only if the two conditions $N_{F} \ll 1$ (far field) and $r^{2} \ll\langle r\rangle_{0}^{2} / N_{F}$ (paraxial) are fulfilled. These two inequalities offer a practical test to determine whether the SPA-SFA approach is valid.

Figure 2 shows the comparison of the exact SFA, Eq. (15), the approximated far field, Eq. (20), and the paraxial far field, Eq. (22), representations of the wave function. The plots correspond to the real part of the wave function along the field polarization axis $(z)$ for an electron ionized at the maximum (a)

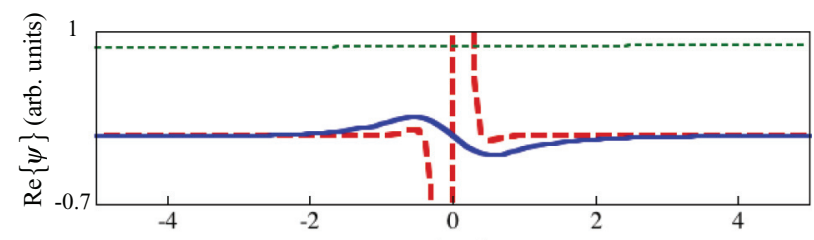

(b)

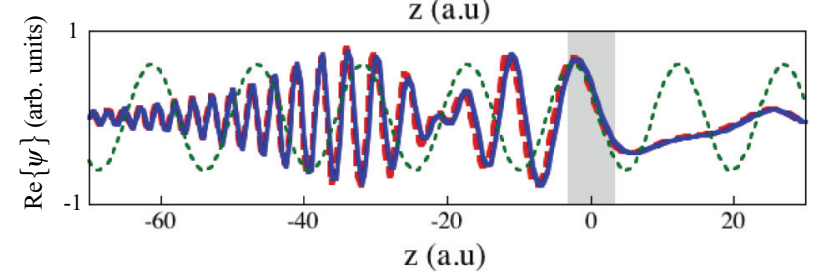

(c)
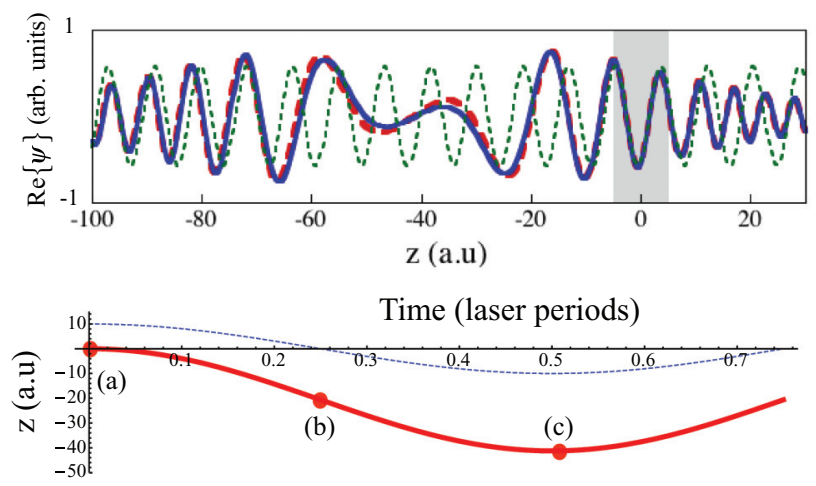

FIG. 2. (Color online) Real part of the wave function $\delta \tilde{\psi}\left(t_{i} ; \mathbf{r}, t\right)$ along the $z$ axis (a) right after ionization $\left(t \simeq t_{i}\right)$, (b) a quarter of cycle after ionization $\left(t-t_{i}=0.25 T, T\right.$ being the laser period), and (c) half cycle after ionization $\left(t-t_{i}=0.5 T\right)$. We choose the ionization time $t_{i}$ corresponding to the maximum amplitude of the electromagnetic field. The exact SFA wave function, Eq. (15), is plotted using a solid blue (gray) line; the far-field approximation, Eq. (20), is plotted using a thick red (gray) dashed line; and the saddle-point-approximated wave function, Eq. (22), is plotted using a thin green (gray) dashed line. The shadowed box shows the spatial region where the saddlepoint-approximated wave function can be used; its limits are defined as $r^{2}=\langle r\rangle_{0}^{2} / N_{F} / 16$. Part (d) of the figure shows the trajectory of a classical electron ionized at $t_{i}$ with velocity $v\left(t_{i}\right)=0$ (solid red (gray) line) and the amplitude of the electric field in arbitrary units (thin dashed blue (gray) line). The red (gray) circles highlight the position of the classical electron at the time instants where times (a), (b), and (c) are evaluated.

of the electric field (a) right after ionization, (b) a quarter of cycle after ionization, and (c) a half-cycle after ionization. The initial bound state is the hydrogen $1 s$, and the field is assumed to be of constant amplitude with a wavelength of $800 \mathrm{~nm}$ (period $\sim 110 \mathrm{au}$ ) and intensity $\sim 1.6 \times 10^{14} \mathrm{~W} / \mathrm{cm}^{2}$. Taking $\langle r\rangle_{0}=2.5$ au [the average radius of $\left.\xi\left(\mathbf{r}_{i}, t_{i}\right)\right]$, the condition $N_{F} \sim 1$ leads to $t-t_{i} \sim 1$, and therefore the far-field regime is reached almost instantaneously after ionization. In this case, we should expect the far-field wave function (20) to be a good approximation to the exact ionized SFA wave function at practically all excursion times. This can be checked in Figs. 2(b) and 2(c), where the far-field-approximated wave function (red [gray] dashed line) reproduces faithfully the exact wave function (solid blue [gray] line). The paraxial approximation breaks, however, for very small excursion times (less than 1 au in this case), as depicted in Fig. 2(a). Figure 2 
also shows the far-field paraxial wave functions (equivalent to the SFA-SPA result) for the three cases (green [gray] dashed lines), which correspond always to plane-wave Volkov solutions. As expected from the above discussion, SFA-SPA is only accurate near the ionization spot, where the paraxial condition is fulfilled. We show as shadowed areas the region enclosed by paraxial condition $r^{2}=\langle r\rangle_{0}^{2} / N_{F} / 16$ which, in our experience, gives a sufficiently precise definition to the upper paraxial limit $r^{2} \ll\langle r\rangle_{0}^{2} / N_{F}$ stated above.

\section{HARMONIC GENERATION}

We now apply our results to the computation of harmonic generation. In semiclassical optics, the radiation of a charge can be computed from the mean dipole acceleration, using Larmor's formula [10]. Therefore, up to constants, the harmonic radiation is described by the dipole acceleration spectrum. Considering the SFA decomposition of the total wave function as the sum of the ground and the continuum $\left|\phi_{0}(t)\right\rangle+|\tilde{\psi}(t)\rangle$, as done in Sec. II, the higher frequency part of the complex acceleration spectrum is given by the most energetic transitions (i.e., continuum bound)

$$
\mathbf{a}(t)=\left\langle\phi_{0}(t)|\hat{\mathbf{a}}| \tilde{\psi}(t)\right\rangle,
$$

where $\hat{\mathbf{a}}=(-1 / m) \nabla V_{c}$ ( $V_{c}$ being the coulomb potential energy), $|\tilde{\psi}(t)\rangle$ is the SFA continuum wave function given by Eq. (5), and $\left|\phi_{0}(t)\right\rangle$ is the initial bound state, evolving field-free, as given by Eq. (2). Using Eqs. (6) and (7) we can rewrite the total acceleration (23) as the sum of elementary contributions corresponding to the wave packets ionized at different times:

$$
\mathbf{a}(t)=\sum_{t_{i}} \delta \mathbf{a}\left(t, t_{i}\right),
$$

where

$$
\begin{aligned}
\delta \mathbf{a}\left(t, t_{i}\right) \equiv & \left\langle\phi_{0}(t)|\hat{\mathbf{a}}| \delta \tilde{\psi}\left(t_{i} ; t\right)\right\rangle=\left(\frac{m}{2 \pi i \hbar} \frac{1}{t-t_{i}}\right)^{3 / 2} \\
& \times \iint \boldsymbol{\alpha}^{*}(\mathbf{r}, t) e^{\frac{i}{\hbar} S_{c l}\left(\mathbf{r}, t, \mathbf{r}_{i}, t_{i}\right)} \xi\left(\mathbf{r}_{i}, t_{i}\right) d \mathbf{r} d \mathbf{r}_{i} \Delta t_{i}
\end{aligned}
$$

here $\boldsymbol{\alpha}(\mathbf{r}, t)=\hat{\mathbf{a}} \phi_{0}(\mathbf{r}, t)$ is the acceleration matrix element in coordinate space. As we have seen above, for the usual laser wavelengths employed in strong-field interactions, Eq. (19) leads to $N_{F} \ll 1$ for all the relevant excursion times in the atomic case. Therefore HHG in atoms is well described in the far-field approximation. In addition, since harmonics are generated near the parent ion, $\boldsymbol{\alpha}(\mathbf{r}, t)$ is only relevant in the neighborhood of the bound-state wave function $\left(|\mathbf{r}| \sim\langle r\rangle_{0}\right)$, thus fulfilling also the paraxial condition. Therefore, the atomic HHG problem can be solved using the far-field paraxial wave function $\delta \tilde{\psi}_{\text {SPA }}$. Insertion of Eq. (22) into Eq. (25) and use of Eq. (24) leads to

$$
\begin{aligned}
\mathbf{a}_{\mathrm{SPA}}(t)= & \int_{t_{0}}^{t}\left(\frac{m}{i \hbar} \frac{2 \pi}{t-t_{i}}\right)^{3 / 2} e^{-\frac{i}{2 m \hbar} \int_{t_{i}}^{t} \pi_{\text {loop }}^{2}\left(t, t_{i}, \tau\right) d \tau} \\
& \times \overline{\boldsymbol{\alpha}}^{*}\left[\boldsymbol{\pi}_{\mathrm{loop}}\left(t, t_{i} ; t\right), t\right] \Xi\left[\boldsymbol{\pi}_{\mathrm{loop}}\left(t, t_{i} ; t_{i}\right), t_{i}\right] d t_{i},
\end{aligned}
$$

where

$$
\overline{\boldsymbol{\alpha}}(\boldsymbol{\pi}, t)=\frac{1}{(2 \pi)^{3 / 2}} \int e^{-\frac{i}{\hbar} \pi \cdot \mathbf{r}} \boldsymbol{\alpha}(\mathbf{r}, t) d \mathbf{r} .
$$

Equation (26) corresponds to the standard saddle-point formula for computing harmonic generation, proposed first by Lewenstein et al. [4], though here it is derived using the dipole acceleration instead of the dipole operator.

We now test our results in the dissociating hydrogen ion molecule $\mathrm{H}_{2}^{+}$. This is a particularly simple system, involving one electron and with cylindrical symmetry if the laser in polarized along the molecular axis, and therefore it has been used as a model for intense field physics in nonatomic systems since the early days [11]. In the present case, we are interested in monitoring the changes in the high-order harmonic spectrum with increasing internuclear distance, in particular in seeing what happens when the condition $N_{F} \ll 1$ is violated. For this, we compare the results for the acceleration spectrum from the exact SFA wave function, Eq. (25), and the saddle-point approximation, Eq. (26). While Eq. (26) is simple enough to be implemented numerically in an efficient way, the exact SFA acceleration (25) requires a double integral in space. The computation, however, can be carried out efficiently following the technique published in Ref. [12], adapted to the length gauge. It is necessary to mention that molecular SFA calculations in the length gauge should include a correction for the space-dependent Stark shift of the initial bound state [13].

The dissociating $\mathrm{H}_{2}^{+}$molecule is modeled by a two-well hydrogenic potential

$$
V_{c}=-\frac{q^{2}}{|r-\mathbf{R} / 2|}-\frac{q^{2}}{|r+\mathbf{R} / 2|},
$$

where $\mathbf{R}$ is the internuclear vector and $q$ is the electron charge. For the case of sufficiently large internuclear distances, the ground state of $\mathrm{H}_{2}^{+}$can be well described by the linear combinations of two hydrogen 1 s orbitals, $\phi_{0}^{H}$,

$$
\phi_{0}^{H_{2}^{+}}(\mathbf{r})=\frac{1}{\sqrt{2}}\left[\phi_{0}^{H}(\mathbf{r}-\mathbf{R} / 2)+\phi_{0}^{H}(\mathbf{r}+\mathbf{R} / 2)\right] .
$$

To estimate the value of the Fresnel number $N_{F}$, we use $R$ as the typical size of the ground-state wave function, i.e., $\langle r\rangle_{0} \simeq R / 2$.

It is known that the high-order-harmonic spectra maps the recollision energy of the semiclassical electron trajectories [14]. To a good approximation, each harmonic is emitted by two different trajectories, named short and long [15], according to their excursion time in the continuum. Generally, the main contribution to the harmonic radiation comes from the short trajectories that are dispersed in the continuum over a smaller time and, therefore, they have a larger recollision probability. Since the Fresnel number $N_{F}$ depends on the excursion time, each harmonic in the spectrum has its own associated $N_{F}$ depending on the excursion time of the associated path.

The left column in Fig. 3 shows some comparisons of the SFA spectra, Fourier transform of Eq. (25), and the SFA-SPA spectra, Fourier transform (26), for the hydrogen atom (first row) and for the $\mathrm{H}_{2}^{+}$molecule at internuclear distances $R=10,20$, and 60 a.u. in the subsequent rows, for a four-cycle laser pulse of $\lambda=800 \mathrm{~nm}$ and intensity $1.6 \times 10^{14} \mathrm{~W} / \mathrm{cm}^{2}$. 
(a)

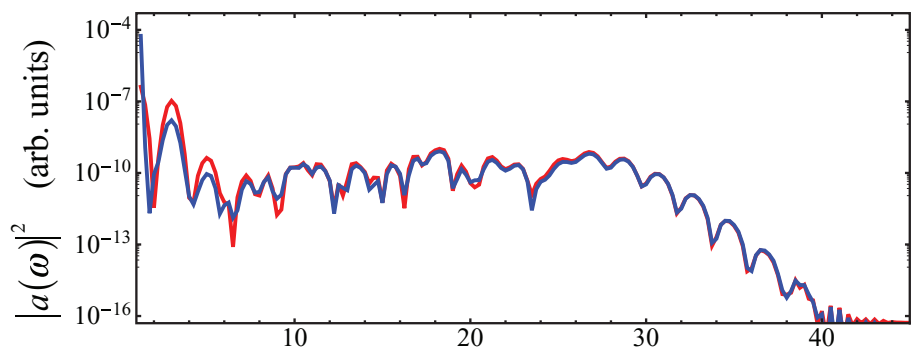

(b)

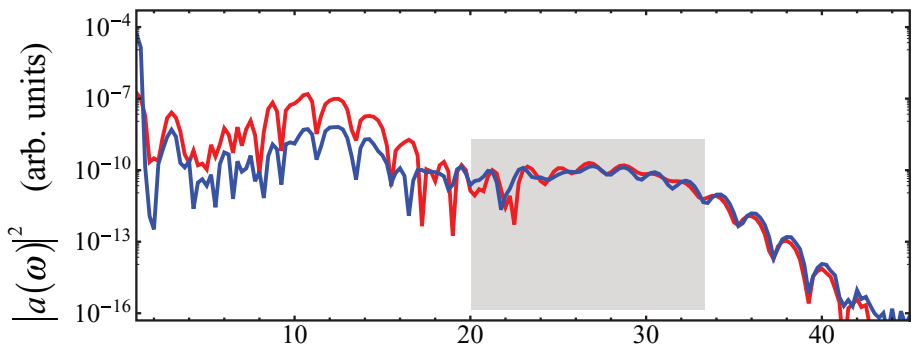

(c)

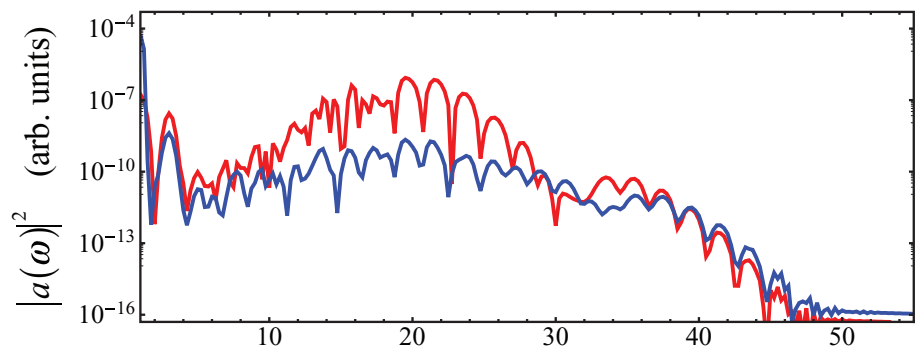

(d)

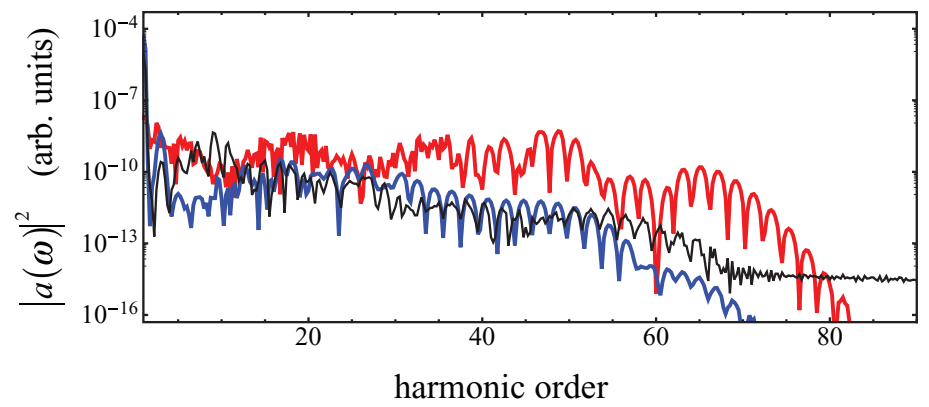

(e)

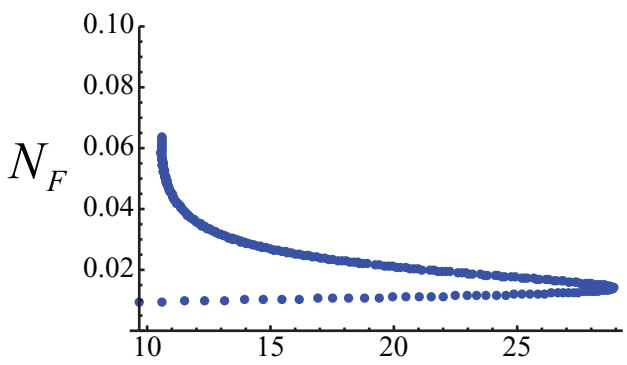

(f)

(g)

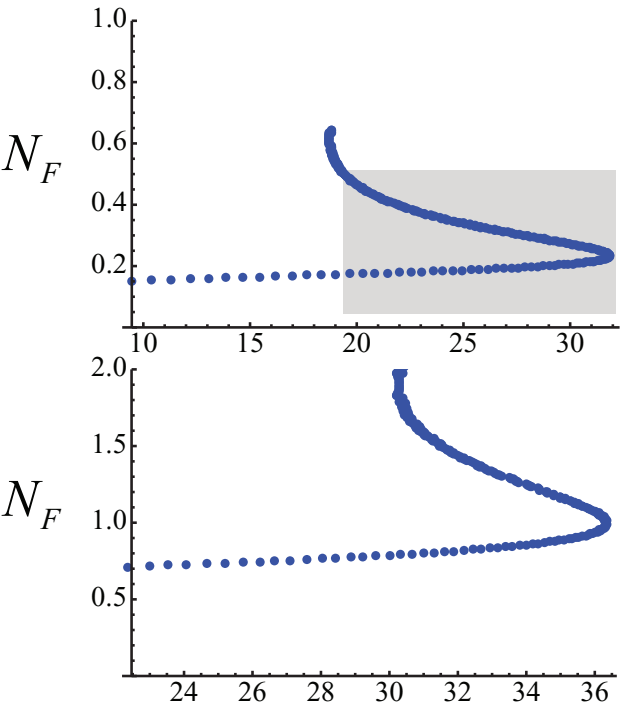

(h)

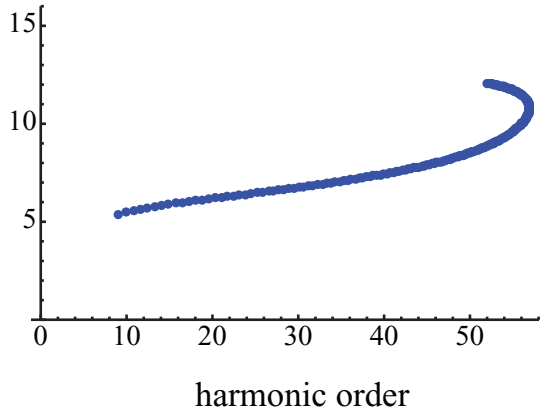

FIG. 3. (Color online) Left column: High-harmonic spectra for (a) a hydrogen atom and (b)-(d) the dissociating $H_{2}^{+}$molecule with internuclear distances 10, 20, and 60 a.u. The Fourier transform of the exact SFA formula (25) are plotted using blue (gray) lines, the SFA-SPA spectra from Eq. (26) are plotted in red (gray), and the results of the exact solution of the Schrödinger equation are plotted in black [only shown in panel (d)]. Right column: Fresnel number $N_{F}$ computed using the excursion times of the classical electron trajectories responsible for the emission of the harmonics. The laser field has a $\sin ^{2}$ four-cycle envelope, 800 -nm wavelength, and intensity $1.6 \times 10^{14} \mathrm{~W} / \mathrm{cm}^{2}$.

SFA results are plotted in blue online, and those of SFA-SPA are in red online. We have also plotted the results of the exact TDSE calculation for the $R=60$ a.u. case, shown as a thin black line in Fig. 3(d). It becomes quite evident that the exact SFA approaches the TDSE results, while the SFA-SPA departs considerably. We note, however, that the exact SFA spectrum does not reproduce the TDSE results with equal quality as in the single-atom case. It is known that Coulomb effects become increasingly important for extended molecules, thus requiring corrections to the standard SFA formulation, beyond the scope of this study [16]. The extension of the spectral cutoff to higher frequencies when increasing the internuclear distance is a well-known effect [17] that is attributed to trajectories initiating on one potential well that rescatter with other [18] (so-called transfer electrons).
As expected from the above discussion, the SPA approach becomes progressively inaccurate as the size of the system increases. The failure of the standard SPA to reproduce extended molecules has been reported before, and it is known to require modifications to the SPA method to include the contribution of the transfer electrons [19]. It is interesting to note, however, that the departure of SPA from the exact SFA is not the same at all harmonics. As an example, Fig. 3(b) shows how the SPA can be still accurate for the higher part of the harmonic spectrum, while being inaccurate at lower frequencies. We find a reason for this in the different excursion times connected to the production of harmonics at different frequencies: Figures 3(e) and 3(f) show $N_{F}$ computed using the excursion times of the classical trajectories that lead to each harmonic. As commented above, every harmonic has 
two trajectory contributions, short and long; therefore each harmonic is associated to two different values of $N_{F}$. Shorter trajectories correspond to the higher $N_{F}$ branch in each of the plots. Since these trajectories generate harmonics more efficiently, our discussion is restricted to the upper branches of the $N_{F}$ plots. If we assume that $N_{F}<0.5$ is already enough to fulfill the condition $N_{F} \ll 1$, we see that Figs. $3(\mathrm{~g})$ and $3(\mathrm{~h})$ predict the failure of the SPA approach for the whole harmonic spectrum [as shown in Figs. 3(c) and 3(d), respectively]. This is not the case for plots in Figs. 3(a) and 3(e), and Figs. 3(b) and 3(f), corresponding to a shorter internuclear distance ( $R=10$ a.u.) and for the single atom. The atomic dimensions are small enough to ensure $N_{F} \ll 1$ at all harmonic frequencies; therefore the SFA-SPA becomes almost exact. Besides, Figs. 3(b) and 3(f) show the interesting regime in which $N_{F}<0.5$ only for the spectral window above the 20th harmonic order. According to this, the SFA-SPA should approach the exact SFA result for harmonics above this threshold, while being inaccurate for the lower order ones. We have shadowed the area for which the far-field condition is fulfilled, showing clearly the correspondence of the validity of the SFA approach with the far-field condition.

\section{CONCLUSIONS}

We have presented a position-space approach to strong-field interactions. By exploiting the analogy with optical scalar diffraction theory, we have been able to determine different regimes in terms of the excursion time of the ionized electron. For long excursion times, the optical-far field analogy allows us to propose an approximated form for the ionized wave function. The standard formulation (based in the strong-field and saddle-point approximations) is found to be a special case of the far-field wave function, restricted to the spatial region near the ionization spot. We develop also the spatial expressions to compute high-order harmonic generation and demonstrate that the standard saddle-point formulation is only valid for small systems. Finally, we use the dissociating $\mathrm{H}_{2}^{+}$ molecule as a test playground to demonstrate our results.

\section{ACKNOWLEDGMENTS}

We are very grateful to A. Benseny and E. Conejero-Jarque for fruitful discussions. We acknowledge support from Spanish MINECO through the research project FIS2009-09522; Junta de Castilla y León, through the research project SA116U13; and a grant from the European Research Council (ERC-2011AdG-291561-HELIOS).

\section{APPENDIX A: COMPUTATION OF THE SPACE-TIME IONIZED WAVEFUNCTION}

The space-time form (5) of the perturbed wave function can be found projecting the ket $|\tilde{\psi}(t)\rangle$ on the space basis $|\mathbf{r}\rangle$. Using $\int d \mathbf{r}_{i}\left|\mathbf{r}_{i}\right\rangle\left\langle\mathbf{r}_{i}\right|=\mathbb{1}$, we can rewrite Eq. (4) as

$$
\tilde{\psi}(\mathbf{r}, t)=\frac{1}{\hbar} \int_{t_{0}}^{t} \int G_{F}^{+}\left(\mathbf{r}, t, \mathbf{r}_{i}, t_{i}\right)\left\langle\mathbf{r}_{i}\left|V_{F}\left(t_{i}\right)\right| \phi_{0}\left(t_{i}\right)\right\rangle d \mathbf{r}_{i} d t_{i},
$$

where we have defined $G_{F}^{+}\left(\mathbf{r}, t, \mathbf{r}_{i}, t_{i}\right) \equiv\left\langle\mathbf{r}\left|G_{F}^{+}\left(t, t_{i}\right)\right| \mathbf{r}_{i}\right\rangle$ as the space-time form of the Volkov propagator, i.e., the propagator for a free electron in a laser field. The expression for the free electron in the field propagator can be found from its definition in terms of the Volkov waves (exact solutions for the free particle in an oscillating electric field) as $G_{F}^{+}\left(t, t_{i}\right) \equiv$ $-i \int|\boldsymbol{\pi}(\mathbf{k}, t)\rangle\left\langle\boldsymbol{\pi}\left(\mathbf{k}, t_{i}\right)\right| d \mathbf{k}$, with

$$
\langle\mathbf{r} \mid \boldsymbol{\pi}(\mathbf{k}, t)\rangle=\frac{1}{(2 \pi)^{3 / 2}} e^{-\frac{i}{2 m \hbar} \int_{t_{0}}^{t} \pi^{2}(\mathbf{k}, \tau) d \tau} e^{i \frac{1}{\hbar} \pi(\mathbf{k}, t) \cdot \mathbf{r}},
$$

where $\boldsymbol{\pi}(\mathbf{k}, \tau)=\hbar \mathbf{k}-(q / c) \mathbf{A}(\tau)$ is the kinetic momentum of the Volkov wave $[\mathbf{A}(\tau)$ being the field vector potential and $\hbar \mathbf{k}$ the canonical momentum]. Therefore

$$
\begin{aligned}
G_{F}^{+}\left(\mathbf{r}, t, \mathbf{r}_{i}, t_{i}\right) & =\int\left\langle\mathbf{r}\left|G_{F}^{+}\left(t, t_{i}\right)\right| \mathbf{r}_{i}\right\rangle d \mathbf{k} \\
& =-\frac{i}{(2 \pi)^{3}} \int e^{\frac{i}{\hbar} S\left(\mathbf{k}, \mathbf{r}, t, \mathbf{r}_{i}, t_{i}\right)} d \mathbf{k},
\end{aligned}
$$

with

$$
\begin{aligned}
& S\left(\mathbf{k}, \mathbf{r}, t, \mathbf{r}_{i}, t_{i}\right) \\
& \quad=-\frac{1}{2 m} \int_{t_{i}}^{t} \pi^{2}(\mathbf{k}, \tau) d \tau+\pi(\mathbf{k}, t) \cdot \mathbf{r}-\pi\left(\mathbf{k}, t_{i}\right) \cdot \mathbf{r}_{i} .
\end{aligned}
$$

Since $S$ is a quadratic function in $\mathbf{k}$, Eq. (A3) can be evaluated as a Gaussian integral around the minimum of $S\left(\mathbf{k}, \mathbf{r}, t, \mathbf{r}_{i}, t_{i}\right)$ in the $\mathbf{k}$ space, $(\partial S / \partial k)_{\mathbf{k}_{c l}}=0$,

$$
\mathbf{k}_{c l}\left(\mathbf{r}, t, \mathbf{r}_{i}, t_{i}\right)=\frac{1}{\hbar\left(t-t_{i}\right)}\left[m\left(\mathbf{r}-\mathbf{r}_{i}\right)+\frac{q}{c} \int_{t_{i}}^{t} \mathbf{A}(\tau) d \tau\right] .
$$

Therefore, the Volkov propagator, Eq. (A3), can be finally written as

$$
G_{F}^{+}\left(\mathbf{r}, t, \mathbf{r}_{i}, t_{i}\right)=\left(\frac{i m}{2 \pi \hbar\left(t-t_{i}\right)}\right)^{3 / 2} e^{\frac{i}{\hbar} S_{c l}\left(\mathbf{r}, t, \mathbf{r}_{i}, t_{i}\right)},
$$

where $S_{c l}$ corresponds to $S$ in Eq. (A4) with the replacement of the kinetic momentum $\boldsymbol{\pi}(\mathbf{k}, \tau)$ by $\boldsymbol{\pi}_{c l}\left(\mathbf{r}, t, \mathbf{r}_{i}, t_{i}, ; \tau\right)$ defined as

$$
\pi_{c l}\left(\mathbf{r}, t, \mathbf{r}_{i}, t_{i} ; \tau\right) \equiv \hbar \mathbf{k}_{c l}\left(\mathbf{r}, t, \mathbf{r}_{i}, t_{i}\right)-\frac{q}{c} \mathbf{A}(\tau),
$$

leading to Eq. (11). Since the combination of Eqs. (A5) and (A7) leads to $\mathbf{r}-\mathbf{r}_{i}=(1 / m) \int_{t_{0}}^{t} \boldsymbol{\pi}_{c l}\left(\mathbf{r}, t, \mathbf{r}_{i}, t_{i}, \tau\right) d \tau, \boldsymbol{\pi}_{c l}$ is the kinetic momentum of the free electron in the field classical trajectory starting at $\left(\mathbf{r}_{i}, t_{i}\right)$ and crossing $\mathbf{r}$ at time $t$. The trajectory at any intermediate time $\tau$ is given by Eq. (10).

Note also that we can rewrite $S_{c l}$ as

$$
S_{c l}\left(\mathbf{r}, t, \mathbf{r}_{i}, t_{i}\right)=\int_{t_{i}}^{t} L\left(\mathbf{r}, t, \mathbf{r}_{i}, t_{i}, \tau\right) d \tau
$$

where $L=\pi_{c l} \cdot \dot{\eta}-H_{F}$ is the Lagrangian. Therefore, $S_{c l}$ corresponds to the action associated with the classical trajectory $\eta_{c l}$. Note that in the context of intense-field physics, developed in the momentum space and saddle-point approximation, the quantity usually termed as action traditionally corresponds to the accumulated kinetic energy $\int_{t_{i}}^{t} \boldsymbol{\pi}^{2}(\mathbf{k}, \tau) d \tau /(2 m)$ and not $S_{c l}$.

By inserting Eq. (A6) into Eq. (A1), we are led to the exact general form for the SFA wave function in space-time (5). This is the well-known Feynman's result [20]: If $S\left(\mathbf{k}, \mathbf{r}, t, \mathbf{r}_{i}, t_{i}\right)$ is a quadratic function, the propagation of the wave function 
between two points in space-time is described exactly using the corresponding classical trajectory.

\section{APPENDIX B: IONIZED WAVE FUNCTION USING THE SADDLE-POINT APPROXIMATION IN MOMENTUM SPACE}

We start with Eq. (4), and by inserting the identity operator $\hat{I}=\int\left|\boldsymbol{\pi}\left(\mathbf{k}, t_{i}\right)\right\rangle\left\langle\boldsymbol{\pi}\left(\mathbf{k}, t_{i}\right)\right| d \mathbf{k}$ and using $|\boldsymbol{\pi}(\mathbf{k}, t)\rangle=$ $i G_{F}^{+}\left(t, t_{i}\right)\left|\boldsymbol{\pi}\left(\mathbf{k}, t_{i}\right)\right\rangle$, we have

$$
|\tilde{\psi}(t)\rangle=\frac{1}{i \hbar} \int_{t_{0}}^{t} \int|\boldsymbol{\pi}(\mathbf{k}, t)\rangle\left\langle\boldsymbol{\pi}\left(\mathbf{k}, t_{i}\right)\left|V_{F}\left(t_{i}\right)\right| \phi_{0}\left(t_{i}\right)\right\rangle d \mathbf{k} d t_{i} .
$$

Therefore, the contribution to $|\tilde{\psi}(t)\rangle$ of the amplitude of the electron ionized at the differential time interval from $t_{i}$ to $t_{i}+\Delta t_{i}$ is

$\delta\left|\tilde{\psi}\left(t_{i} ; t\right)\right\rangle=\frac{\Delta t_{i}}{i \hbar} \int|\boldsymbol{\pi}(\mathbf{k}, t)\rangle\left\langle\boldsymbol{\pi}\left(\mathbf{k}, t_{i}\right)\left|V_{F}\left(t_{i}\right)\right| \phi_{0}\left(t_{i}\right)\right\rangle d \mathbf{k} . \quad$ (B2)
By projecting on the spatial coordinates $|\mathbf{r}\rangle$ and using Eq. (A2), we have

$$
\begin{aligned}
\delta \tilde{\psi}\left(t_{i} ; \mathbf{r}, t\right)= & \frac{\Delta t_{i}}{(2 \pi)^{3 / 2}} \int e^{-\frac{i}{2 m \hbar} \int_{t_{i}}^{t} \pi^{2}(\mathbf{k}, \tau) d \tau} e^{i \frac{1}{\hbar} \pi(\mathbf{k}, t) \cdot \mathbf{r}} \Xi \\
& \times\left[\boldsymbol{\pi}\left(\mathbf{k}, t_{i}\right), t_{i}\right] d \mathbf{k},
\end{aligned}
$$

where we have used Eqs. (8) and (21). Following the standard procedure [4], Eq. (B3) is computed at the saddle point in momentum space of the integral over the kinetic energy. The momentum of the saddle point corresponds to $\pi_{\text {loop }}\left(t, t_{i} ; \tau\right)$; therefore

$$
\begin{aligned}
\delta \tilde{\psi}\left(t_{i} ; \mathbf{r}, t\right)= & \frac{\Delta t_{i}}{(2 \pi)^{3 / 2}} e^{i \frac{1}{\hbar} \pi_{\text {loop }}\left(t, t_{i} ; \tau\right) \cdot \mathbf{r}} \Xi\left[\boldsymbol{\pi}_{\text {loop }}\left(t, t_{i} ; \tau\right), t_{i}\right] \\
& \times \int e^{-\frac{i}{2 m \hbar} \int_{t_{i}}^{t} \pi^{2}(\mathbf{k}, \tau) d \tau} d \mathbf{k}
\end{aligned}
$$

Equation (22) results after the Gaussian integration.
[1] L. V. Keldysh, Zh. Eksp. Teor. Fiz. 47, 1945 (1964) [Sov. Phys. JETP 20, 1307 (1965)].

[2] F. H. M. Faisal, J. Phys. B: At. Mol. Phys. 6, L89 (1973).

[3] H. R. Reiss, Phys. Rev. A 22, 1786 (1980).

[4] M. Lewenstein, P. Balcou, M. Y. Ivanov, A. L'Huillier, and P. B. Corkum, Phys. Rev. A 49, 2117 (1994).

[5] P. Salières et al., Science 292, 902 (2001).

[6] M. D. Volkov, Z. Phys. 94, 250 (1935).

[7] O. I. Tolstikhin and T. Morishita, Phys. Rev. A 86, 043417 (2012).

[8] M. Born and E. Wolf, Principles of Optics (Cambridge University Press, Cambridge, 1999).

[9] A. D. Bandrauk, S. Chelkowski, and K. J. Yuan, IRAMP 2, 1 (2011).

[10] J. D. Jackson, Classical Electrodynamics (Wiley, New York, 1998).

[11] T. Zuo, S. Chelkowski, and A. D. Bandrauk, Phys. Rev. A 48, 3837 (1993).
[12] J. A. Pérez-Hernández, L. Roso, and L. Plaja, Opt. Express 17, 9891 (2009).

[13] O. Smirnova, M. Spanner, and M. Ivanov, J. Mod. Opt. 54, 1019 (2007).

[14] P. B. Corkum, Phys. Rev. Lett. 71, 1994 (1993).

[15] M. Lewenstein, P. Salières, and A. L'Huillier, Phys. Rev. A 52, 4747 (1995).

[16] M. F. Ciappina, C. C. Chirilă, and M. Lein, Phys. Rev. A 75, 043405 (2007).

[17] R. Numico, P. Moreno, L. Plaja, and L. Roso, J. Phys. B: At. Mol. Opt. Phys. 31, 4163 (1998).

[18] P. Moreno, L. Plaja, and L. Roso, Europhys. Lett. 28, 629 (1994).

[19] C. C. Chirilă and M. Lein, Phys. Rev. A 73, 023410 (2006).

[20] R. P. Feynman and A. R. Hibbs, Quantum Mechanics and Path Integrals (McGraw-Hill, New York, 1965). 